\title{
DOS À LA MER ?
}

Les pays européens du Sud face à l'immigration

Évelyne Ritaine

Presses de Sciences Po | « Critique internationale »

2003/1 n $\mathrm{n}^{0} 18 \mid$ pages 143 à 158

ISSN 1290-7839

ISBN 2724629698

\section{Article disponible en ligne à l'adresse :}

https://www.cairn.info/revue-critique-internationale-2003-1-page-143.htm

Distribution électronique Cairn.info pour Presses de Sciences Po.

(C) Presses de Sciences Po. Tous droits réservés pour tous pays.

La reproduction ou représentation de cet article, notamment par photocopie, n'est autorisée que dans les limites des conditions générales d'utilisation du site ou, le cas échéant, des conditions générales de la licence souscrite par votre établissement. Toute autre reproduction ou représentation, en tout ou partie, sous quelque forme et de quelque manière que ce soit, est interdite sauf accord préalable et écrit de l'éditeur, en dehors des cas prévus par la législation en vigueur en France. Il est précisé que son stockage dans une base de données est également interdit. 


\section{Dos à la mer?}

\section{Les pays européens du Sud face à l'immigration}

Presses de Sc. Po. | Critique internationale

$$
\begin{aligned}
& \text { 2003/1 - no } 18 \\
& \text { pages } 143 \text { à } 158 \\
& \text { ISSN 1290-7839 }
\end{aligned}
$$

Article disponible en ligne à l'adresse:

http://www.cairn.info/revue-critique-internationale-2003-1-page-143.htm

\section{Pour citer cet article :}

"Dos à la mer ? " Les pays européens du Sud face à l'immigration, Critique internationale, 2003/1 no 18, p. $143-158$.

Distribution électronique Cairn.info pour Presses de Sc. Po..

(c) Presses de Sc. Po.. Tous droits réservés pour tous pays.

La reproduction ou représentation de cet article, notamment par photocopie, n'est autorisée que dans les limites des conditions générales d'utilisation du site ou, le cas échéant, des conditions générales de la licence souscrite par votre établissement. Toute autre reproduction ou représentation, en tout ou partie, sous quelque forme et de quelque manière que ce soit, est interdite sauf accord préalable et écrit de l'éditeur, en dehors des cas prévus par la législation en vigueur en France. II est précisé que son stockage dans une base de données est également interdit. 


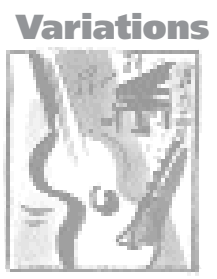

\title{
Dos à la mer?
}

\author{
Les pays européens du Sud face à l'immigration
}

\author{
par Évelyne Ritaine
}

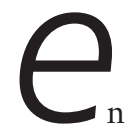

n Méditerranée, certains villages côtiers, bâtis à l'écart des flots, tournent le dos à la mer. Jadis, on y fuyait la malaria endémique des côtes ; on y était berger ou paysan, jamais pêcheur ; on y redoutait, surtout, les invasions par la mer. Il est troublant de voir certains pays d'Europe du Sud renouer avec cette ancestrale méfiance depuis qu'ils connaissent le développement d'une immigration nouvelle pour eux, et largement méditerranéenne : ils sont saisis par le syndrome de l'invasion, qu'il vaudrait sans doute mieux, au regard des chiffres, désigner comme fantasme1. N'ont-ils pas été, l'Italie et l'Espagne surtout, parmi les États les plus militants, durant la présidence espagnole de l'Union européenne, pour que les instances européennes mettent en œuvre une politique d'immigration répressive ? Pourtant l'édification d'une « Europe forteresse » pourrait bien couper ces pays de leur

\footnotetext{
1. En Italie, l'immigration régulière représente 2,9\% de la population totale en 2001 ; les groupes les plus importants viennent du Maroc (158 000 personnes), d'Albanie (144 000), de Roumanie (75 000), des Philippines (64 000), de Chine (57 000) (Caritas Italia, Anticipazioni Dossier statistico immigrazione 2002). En Espagne, l'immigration régulière représente 2,5\% de la population totale en 2001 ; les principales origines sont le Maroc (215 950), l'Équateur (56 634), la Chine (31 602), la Colombie (31 520) (Ministerio del Interior). Au Portugal, l'immigration régulière représente 1,9\% de la population totale ; les groupes les plus importants sont les Cap-Verdiens (56 274), les Ukrainiens (52 578), les Brésiliens (51 449), les Angolais (26 074) (Serviço de Estrangeiros e Fronteiras, 2002). 
histoire méditerranéenne et les séparer de leurs principaux partenaires méridionaux.

La méfiance n'y est pas socialement innée cependant. Longtemps l'immigration croissante a été silencieusement absorbée par l'économie souterraine, régulée par des arrangements sociaux locaux plus ou moins heureux. La méfiance, et parfois le rejet xénophobe, y sont en fait des constructions politiques. C'est le lent travail politique de certains partis qui a imposé l'interprétation de l'immigration comme risque sécuritaire, à l'exclusion de toute autre dimension, en Italie et en Espagne. Dans des stratégies de conquête du pouvoir ou de renforcement de la puissance étatique, le phénomène migratoire est instrumentalisé à des fins de politique intérieure, de façon d'autant plus prégnante que le contrôle des instances gouvernementales par ces partis institutionnalise les représentations sécuritaires dans des politiques d'État. S'agit-il là d'une forme d'autisme politique?

L'interprétation sécuritaire n'apparaît cependant possible que dans certaines conditions. Conditions de fragmentation politique, qui nécessitent de la part des nouveaux acteurs politiques des discours fortement distinctifs, certes. Conditions de crise politique, qui mettent à mal les catégories classiques du politique et favorisent la montée de tous les thèmes de l'antipolitique, certes. Mais aussi conditions discursives : le contre-exemple du Portugal où, jusqu'à présent, la thématique sécuritaire peine à s'imposer, atteste que l'existence d'un discours politique consensuel et anciennement institué sur la question migratoire peut bloquer l'exploitation populiste du thème. La comparaison des trois cas nationaux donne ainsi à penser que l'hégémonie de l'interprétation sécuritaire de l'immigration signe peutêtre une incapacité discursive plus profonde, quant au consensus national et aux règles publiques.

Une telle dérive sécuritaire fait courir à ces pays le risque de perdre les ressources diplomatiques et politiques liées à leur enracinement méditerranéen. Leurs rapports avec leurs voisins immédiats sont désormais placés quasi exclusivement sous le signe de rapports de force, policiers et économiques. Ils perdent peu à peu leur fonction implicite de médiateurs vis-à-vis des pays de culture musulmane, pris qu'ils sont dans la montée d'une opinion anti-islamique. Ils apparaissent désormais comme plus européens que méditerranéens : dos à la mer ?

\section{Le temps d'une non-politique}

Les pays d'Europe du Sud ne sont devenus pays d'immigration qu'insensiblement. Ce n'est qu'à partir des années soixante-dix, lorsque les traditionnels importateurs de main-d'œuvre d'Europe de l'Ouest ont fermé leurs frontières à l'immigration économique, que les migrants ont commencé à entrer en masse par le sud de l'Europe, d'abord dans l'espoir de gagner par la suite les zones les plus riches, puis peu à peu en se résignant à y demeurer. Longtemps, ce développement de 
l'immigration s'est fait à bas bruit. Parce que le phénomène n'a jamais été quantitativement important, par comparaison avec les grands pays d'immigration. Parce que ces sociétés méridionales sont travaillées par de multiples arrangements sociaux, et que ceux de l'économie souterraine trouvent leur compte au développement d'une main-d'œuvre informelle. Ainsi les scénarios de l'intégration du travail immigré au marché du travail sont-ils assez semblables dans les trois cas $^{2}$ : la main-d'œuvre immigrée occupe les emplois dont ne veulent plus les travailleurs nationaux, désormais mieux formés et plus exigeants ; les immigrants les plus récents et les plus précaires sont employés comme saisonniers dans l'agriculture intensive (Mezzogiorno italien, Andalousie) ou comme manœuvres dans les travaux publics et le bâtiment (Lisbonne, les grandes villes italiennes et espagnoles) ; lorsqu'ils se sont stabilisés, les travailleurs immigrés tentent de s'insérer dans le marché du travail des zones plus industrialisées (Italie du Nord, Barcelone, Madrid).

Ce n'est qu'au début des années quatre-vingt-dix que l'immigration commença à apparaître comme un problème public, encore que gérable, à ce moment-là, par des mesures de politique publique classique. Leur absence avait en effet laissé se développer des zones de ghetto où la présence immigrée était à la fois concentrée et précarisée. Des ONG, la presse, certains politiques s'émurent alors des conditions de vie désastreuses dans les ghettos de Villa Literno près de Naples, de la Pantanella à Rome, du centre-ville à Gênes, des campagnes andalouses ou des barracas des faubourgs de Lisbonne. D'autres acteurs entrèrent dans des attitudes de rejet, qui pouvaient aller de la formation de comités de défense de quartier à l'éclatement de graves incidents xénophobes ${ }^{3}$. Cette progressive dramatisation de

\footnotetext{
On ne peut estimer scientifiquement l'importance de l'immigration clandestine, celle-ci étant par nature mouvante. De plus, les estimations sont en elles-mêmes des enjeux politiques, susceptibles de toutes les manipulations. Pour les pays qui nous occupent ici, la prise en compte de l'immigration irrégulière ne changerait pas fondamentalement l'estimation globale (en Italie par exemple, Caritas, une des sources les plus sûres, estime les clandestins à environ 300000 personnes, les immigrés réguliers étant au nombre de 1687 000), qui les place parmi les pays européens où résident le moins d'étrangers : la moyenne de l'immigration régulière dans les États de l'UE est à 5 \% de la population par pays, l'Allemagne, l'Autriche et la Belgique dépassant les 10 \% (OCDE, SOPEMI, 2001).

Cette relativisation n'exonère évidemment pas de la nécessaire prise en compte de l'accentuation de la pression migratoire sur ces territoires (comme sur tout le reste de l'Union européenne), et du caractère souvent dramatique (débarquements sauvages, exodes, noyades, réseaux de trafic de personnes, etc.) que celle-ci prend dans ces pays, caractère facilement exploitable par les médias (Voir A. Dal Lago, « La tautologia della paura », Rassegna italiana di sociologia, XXXX, 1, 1999).

2. Sur ces questions, voir E. Mingione, « Labour market segmentation and informal work in Southern Europe », European Urban and Regional Studies, 2(2), 1995 ; E. Mingione, Fragmented Societies, Oxford, Blackwell, 1991.

3. Cette période est traitée pour chacun des pays dans « Enjeux migratoires en Europe du Sud », Pôle Sud, 11, 1999 (coordination E. Ritaine). Le présent article s'appuie sur une opération de recherche menée au CERVL (Sciences-Po Bordeaux) entre 1999 et 2001 sur la « Construction des enjeux migratoires en Europe du Sud » (Italie, Espagne et Portugal, la Grèce devant faire l'objet de travaux ultérieurs), avec le soutien du CNRS (Programme « L'identité européenne en questions ») et de la région Aquitaine. Ses résultats sont en cours de publication dans E. Ritaine (dir.), Politiques de l'Étranger. L'Europe du Sud face à l'immigration, à paraitre, 2003. Dans la préparation de cet article, les remarques de Célia Barbosa, Béatrice Hibou, Fernando L. Machado et G. Sciortino m'ont été précieuses et je les en remercie.
} 
l'immigration fut accentuée par les conséquences de l'adhésion des trois pays aux accords de Schengen ${ }^{4}$ : au début des années quatre-vingt-dix, l'obligation de visa fut instaurée pour tous les immigrés, le contrôle des frontières et la lutte contre l'immigration clandestine devinrent la lettre, sinon la pratique, des politiques nationales. Avec pour conséquence classique d'accentuer la pression de l'immigration clandestine : les tentatives d'entrée se firent de plus en plus pressantes et périlleuses, les réseaux de passeurs s'organisèrent, les drames liés aux passages clandestins se multiplièrent (naufrages des pateras dans le détroit de Gibraltar et des scaffi en Adriatique, exodes albanais sur les côtes adriatiques). Cette sinistre chronique provoqua la mobilisation de nombreuses $\mathrm{ONG}$, qui entreprirent alors de faire pression sur les politiques pour que soient votées des lois « justes » (pour reprendre l'expression italienne de l'époque) sur le statut des immigrés. Les premières lois promulguées sous cette double pression des accords de Schengen et de la mobilisation associative ${ }^{5}$ furent des lois relativement équilibrées entre nécessité du contrôle et affirmation des droits des immigrés.

Certes, ces lois n'étaient que l'amorce d'un possible cercle vertueux pour des pays dépourvus, à la notable exception du Portugal, de toute expérience en matière d'immigration. Les débats qui les accompagnèrent, la réflexion sur les problèmes que posa leur mise en application permirent aux ONG de se forger une capacité d'expertise et une réelle culture d'intervention en la matière. À l'inverse, les nombreuses résistances de l'appareil étatique (notamment la constante reprise en main répressive par les ministères de l'Intérieur) vidèrent souvent de leur sens les mesures les plus novatrices. Enfin et surtout, là comme ailleurs, deux points d'achoppement des lois d'immigration sont restés non résolus. Celui du contrôle des frontières tout d'abord : le décréter sur le papier ne résout évidemment rien, comme en témoignent le recours récurrent au déploiement des forces armées sur les côtes ou les projets de blindage de plus en plus fous, mais aussi la multiplication des régularisations de masse pour tenter, de loin en loin, d'assainir la situation. Celui du statut politique de l'immigré surtout : même les lois les plus avancées (loi 40/1998 dite loi Turco-Napolitano portée par la majorité de centre-gauche en Italie, et loi organique 4/2000 en Espagne, votée malgré l'opposition du gouvernement du $\mathrm{PP})$ ne parvinrent pas, faute de consensus politique, à élaborer un statut de citoyenneté immigrée.

Au début des années quatre-vingt-dix, il semblait donc que les pays de l'Europe du Sud commençaient un long cheminement en matière de politiques migratoires, entre leur propre maturation politique de la question et la probable européanisation de ce thème. Or, de façon surprenante, en Italie et en Espagne, la question migratoire a surgi très brutalement au centre de l'agenda politique, où elle demeure un enjeu politique de premier plan. 


\section{Une forme d'autisme politique}

Dans ces deux pays, des conjonctures singulières ont entraîné une très forte politisation de la question migratoire, qui s'est trouvée prise dans des jeux de concurrence partisane. Cette instrumentalisation modifie complètement la teneur du débat : la question migratoire devient en quelque sorte une question poreuse, par laquelle les systèmes sociopolitiques parlent d'eux-mêmes beaucoup plus que du phénomène en question ${ }^{6}$. Cette façon de se représenter soi-même en parlant de l'Étranger a fini, comme dans bien d'autres pays européens, par institutionnaliser la thématique sécuritaire : la question, politique, de la présence immigrée s'y est trouvée réduite à une menace sécuritaire.

Il est clair désormais que, face aux différents symptômes de l'incertitude liée à la globalisation, bien des forces politiques en Europe ont développé des stratégies fantasmatiques de désignation de menaces extérieures et de proposition de solutions simplistes 7 . Il s'agit là du cœur même de la tendance néopopuliste qui affecte tant de systèmes politiques ${ }^{8}$. De tous les dangers fantasmés désignés par les discours néopopulistes, celui de l'immigration est le moins coûteux à manipuler : le niveau d'organisation des immigrés est faible, ainsi que les intérêts qui se mobilisent en faveur de leurs droits, et il est relativement aisé de mettre tout le monde d'accord sur le renforcement des contrôles?.

Au-delà de ce malaise commun, l'utilisation stratégique du thème sécuritaire est toujours connotée par les enjeux de la conjoncture nationale dans laquelle elle se déploie : l'interprétation sécuritaire se fait jour partout, elle aboutit à des productions législatives assez semblables - ne serait-ce qu'en raison de l'obligation de respecter les normes communautaires - mais elle n'a pas partout le même sens politique.

\footnotetext{
4. L'accord intergouvernemental de Schengen a été signé le 14 juin 1985 entre l'Allemagne, la Belgique, le Luxembourg, les Pays-Bas et la France. L'Italie y a adhéré en 1990, l'Espagne et le Portugal en 1991, la Grèce en 1992. Il est entré en vigueur le 26 mars 1995, en excluant l'Italie et la Grèce de la mise en application. L'Italie y a été intégrée en 1998, la Grèce en 2000. Les obligations liées à Schengen sont désormais comprises dans le traité d'Amsterdam (2 octobre 1997) qui en prévoit la communautarisation progressive.

5. Pour l'Italie, loi 39/1990 dite loi Martelli ; pour l'Espagne, LOEX 1985 (Ley de Extranjeria) qui anticipait sur l'entrée de l'Espagne dans la Communauté européenne (1986) et sur son adhésion à l'accord de Schengen (1991).

6. C'est la thèse désormais classique de la « fonction-miroir » de l'immigration développée par A. Sayad, La double absence, Paris, Le Seuil, 1999.

7. Z. Bauman, In Search of Politics, Londres, Polity Press, 1999.

8. La littérature sur les formes actuelles de populisme (« néopopulismes », pour les distinguer des formes classiques) insiste à juste titre sur le fait que, si certaines formations politiques d'aujourd'hui peuvent être qualifiées de néopopulistes, des tentations néopopulistes travaillent aussi la plupart des partis. C'est pourquoi le néopopulisme est surtout analysé en termes programmatiques. G. Hermet, Les populismes dans le monde : une bistoire sociologique, XIXe-XXe siècle, Paris, Fayard, 2001 ; Y. Mény, Y. Surel, Par le peuple, pour le peuple. Le populisme et les démocraties, Paris, Fayard, 2000 ; A. Mastropaolo, Antipolitica. All'origine della crisi italiana, Naples, L'Ancora, 2000 ; P. Ignazi, « Lintramontabile fascino del populismo », Il Mulino, 1, 2002. 9. G. Sciortino, L'ambizione della frontiera. Le politiche di controllo migratorio in Europa, Milan, Franco Angeli, 2000.
} 
En Italie, elle est aujourd'hui le fruit d'un processus néopopuliste exemplaire. L'acmé de la profonde crise politique italienne se situe entre 1992 et 1994 : dans ce contexte traumatique, l'électorat était sensible à tous les thèmes de l'État de droit et de l'ordre public ${ }^{10}$. Alors des « entrepreneurs politiques de la crise $»^{11}$, donc de l'incertitude politique, commencèrent à utiliser le thème migratoire dans leurs interprétations de la situation. Le geste instituant fut celui de la Ligue du Nord qui, dès le début de la décennie et avec de plus en plus d'insistance à mesure de l'affaiblissement de son influence, développa une thématique xénophobe violente. Ce parti est en effet une manifestation typique de «populisme d'exclusion $»^{12}$ : sa culture politique est tout entière paranoïaque, alliant l'anti-étatisme (dénonciation du gouvernement central), l'ethnonationalisme (défense de l'intégrité culturelle des communautés localisées), le rejet de l'étranger (dénonciation de l'invasion immigrée). Tout au long des années où les nouveaux partis nés de la crise tentèrent d'accéder au pouvoir, la Ligue se servit d'une thématique xénophobe pour se distinguer de ses alliés ${ }^{13}$. Même si son poids électoral est demeuré modeste, elle contribua ainsi à autoriser un discours xénophobe en Italie, d'autant plus aisément que celui-ci n'empruntait pas les justifications de la hiérarchisation raciale, mais se présentait comme un constat de bon sens, remontant des problèmes quotidiens, vécus par tout un chacun, vers leurs causes désignées (la présence étrangère et l'irresponsabilité des politiques). À partir de 1994 et de la coalition entre Forza Italia, la Ligue du Nord et Alliance nationale, le thème migratoire, qui semblait de plus en plus rentable électoralement, subit une forme d'euphémisation par le travail de la coalition. En effet, Alliance nationale, qui venait à peine de se refaire une virginité en reniant son passé néofasciste, ne pouvait se permettre d'afficher une thématique raciste ou xénophobe : elle s'aligna donc sur une ligne autoritaire et sécuritaire, axée sur la défense de l'ordre public. Opérant dans le court-circuit discursif classique qui lie mécaniquement immigration et délinquance, le parti réussit ainsi à la fois à se positionner comme parti de gouvernement et à promouvoir une position sécuritaire face à l'immigration ${ }^{14}$. Il en allait de même pour Forza Italia, dont la ligne politique est toujours dictée par l'opportunisme électoral : elle se positionna sur une interprétation sécuritaire assez générale, laissant à ses alliés le soin de surenchérir, si besoin était, sur ce thème. Un bon tableau d'ensemble des nuances de la coalition néopopuliste à la fin des années quatre-vingt-dix émerge de leurs réactions à la loi sur l'immigration votée par le centre-gauche en 1998. La Ligue du Nord évoqua le risque d'une «marocchinizzazione » du pays ; Alliance nationale affirma qu'il y avait « escroquerie car cette loi ne répond pas aux exigences de légalité et de sécurité des citoyens italiens »; Forza Italia se contenta de parler d'« un texte démagogique et bâclé » 15 .

L'action politique de ces acteurs, puis leur deuxième accession au pouvoir en 2001, ont contribué à l'institutionnalisation définitive ${ }^{16}$ de l'interprétation sécuritaire. 
Celle-ci, simple à diffuser et fortement explicative, est devenue l'interprétation hégémonique grâce à l'action conjointe du discours politique et de ses relais médiatiques : il est très difficile de lui opposer une autre interprétation ${ }^{17}$. La gauche italienne, toute à sa propre crise interne, n'a pas su, sur ce thème comme sur d'autres, élaborer une interprétation alternative. Elle s'est peu à peu laissée contaminer par l'ambiance sécuritaire, au point que la loi votée sous le gouvernement de centregauche en porte la trace et qu'aucun acteur politique ne cherche à penser le statut politique possible de l'immigré : celui-ci est dorénavant et exclusivement un danger public. La nouvelle loi votée en Italie en août 2002 est, en toute logique, une des plus répressives d'Europe ${ }^{18}$.

La construction de l'interprétation sécuritaire de l'immigration, pour aboutir à des résultats législatifs assez semblables, n'en est pas moins politiquement très différente en Espagne : elle s'inscrit dans un virage autoritaire de l'État central espagnol, sous l'égide du Parti populaire. La politisation de la question y a été plus lente, plus tardive, plus insidieuse aussi. Elle fut surtout placée sous le signe d'une stratégie sécuritaire du gouvernement lui-même. En effet, la question de l'immigration ne fut longtemps - entre l'adhésion à l'accord de Schengen et les nouvelles lois d'immigration entrées en vigueur en 2000 - que faiblement politisée, objet d'un consensus un peu incertain, dominé par le référentiel démocratique de la transition espagnole, et surtout travaillé par les mobilisations associatives ${ }^{19}$. Ce ne fut qu'au tournant des années 2000 qu'une politisation brutale se fit jour. La tension de la conjoncture politique y fut pour beaucoup : le premier gouvernement Aznar prenait fin après une législature compliquée par l'absence de majorité absolue du Parti populaire et la nécessité de construire des majorités ponctuelles avec les

10. E. Ritaine, « Noi e gli altri : l'enjeu migratoire, miroir de la crise politique italienne », Pôle Sud, op. cit. ; voir aussi ma contribution sur l'Italie dans Politiques de l'Étranger, op. cit.

11. I. Diamanti, La Lega : geografia, storia e sociologia di un nuovo soggetto politico, Rome, Donzelli, 1993.

12. H.G. Betz, «Exclusionary populism in Western Europe in the 1990s. Electoral success and political impact », Conférence UNRISD Racism and Public Policy, Durban, sept. 2001, www.unrisd.org ; H.G. Betz, Radical Right-Wing Populism in Western Europe, New York, St. Martin's Press, 1994 ; R. Brubaker, « De l'immigré au citoyen », Actes de la recherche en sciences sociales, 99, 1993.

13. Voir E. Biorcio, La Padania promessa, Milan, Saggiatore, 1997.

14. «Immigrés : AN insiste sur les règles », titrait $I l$ Sole-24 ore, 27 février 2000.

15. Cité par Caritas di Roma, F. Pittau, L’immigrazione alle soglie del 2000, Rome, Sinnos, 1999.

16. Au sens de : extériorisation (par rapport aux acteurs qui l'ont formulée), objectivation et intériorisation généralisée d'une thématique, selon P. Berger et T. Luckmann, La construction sociale de la réalité, Paris, Méridiens Klincksieck, 1986.

17. Processus analysé par A. Dal Lago : «Dans la sociologie de la communication, cette interaction cumulative de facteurs capables de créer une opinion dominante est désignée comme "spirale du silence". Quand une "voix" est progressivement légitimée par les médias, il devient pratiquement impossible, pour les voix différentes, de la contrecarrer ». Il se réfère à E. Noelle-Neuman, Die Schweigespirale, Munich, Piper, 1980 (A. Dal Lago, Non-persone. L'esclusione dei migranti in una società globale, Milan, Feltrinelli, 1999, p. 23).

18. Loi 189/2002, dite loi Bossi-Fini.

19. H. Pérés, « L'Europe commence à Gibraltar : le dilemme espagnol face à la découverte de l’immigration », Pôle Sud, op. cit. 
partis nationalitaires, qui en avaient profité pour développer une surenchère permanente sur leurs propres thématiques. L'enjeu électoral du PP était clairement de revenir au pouvoir avec une majorité absolue.

Or, dans cette période, la question migratoire surgit de façon inattendue sur l'agenda politique. En février 2000, éclataient de violentes émeutes racistes à El Ejido, un des hauts lieux de l'agriculture intensive d'Andalousie ${ }^{20}$. Véritable pogrom contre les travailleurs clandestins marocains, ces événements semblent bien avoir été la réponse locale à une nouvelle loi d'immigration, votée fin 1999, jugée trop libérale et aussi risquant d'entraîner une régularisation dont ne voulaient pas les employeurs locaux : les intérêts de l'économie souterraine pèsent lourd dans cette région, poids auquel la classe politique locale, proche des fractions dures du PP, ne pouvait sans doute pas être insensible ${ }^{21}$. L'autre facteur d'accélération tint à un parcours étrange du nouveau texte de loi. Depuis de longs mois s'était ouvert un intense travail de concertation entre les ONG impliquées dans l'aide aux immigrés, le parti nationalitaire catalan CiU et la frange libérale du PP (dont le ministre du Travail), qui aboutit à la rédaction d'une proposition de loi très libérale sur l'immigration. Le gouvernement Aznar sembla prendre conscience avec retard de ce caractère libéral et tenta en vain de s'opposer au texte, qui fut finalement adopté, faute de majorité contraire, fin 1999, alors que la compétition électorale était déjà ouverte : J.-M. Aznar s'empressa alors de faire de son abrogation une de ses promesses électorales. Cette instrumentalisation correspondait, dans la conjoncture, à un calcul électoral simple. Mais il y avait plus : ayant démontré sa capacité à gouverner démocratiquement, la droite espagnole entendait revenir au pouvoir en affirmant plus fermement ses valeurs centrales, celles de la puissance étatique et de la pérennité de la nation. Son discours insiste désormais sur l'ordre public et sur l'État de droit, en choisissant de réaffirmer le contrôle du territoire par l'État central. Ce tournant se traduit par un programme sécuritaire, en premier lieu dirigé contre le terrorisme basque (le danger intérieur), mais qui englobe aussi la lutte contre l'immigration clandestine (le péril extérieur). Par un biais différent de celui de l'Italie, en Espagne également l'interprétation de l'immigration, lorsqu'elle se politise, se fait sur le mode sécuritaire. Dans ce cas aussi l'immigré est une menace : la victoire électorale du PP, qui a conquis cette fois la majorité absolue, a été suivie de l'immédiate « contre-réforme » de la loi sur l'immigration et de la promulgation d'un texte très répressif ${ }^{22}$. Elle a aussi entraîné une politisation virulente de la question, prise désormais, alors que le calendrier électoral espagnol est chargé d'échéances importantes jusqu'en 2004, dans le jeu complexe des concurrences politiques entre les différents niveaux de gouvernement et de représentation ${ }^{23}$.

Il n'est pas indifférent que, dans ces deux pays, on soit passé d'une situation encore incertaine, entre mobilisations solidaires et épisodes locaux de xénophobie, à une organisation permanente et explicite du rejet : les agences politiques, en fournissant 
un schéma d'explication et de légitimation de celui-ci, ont contribué à l'institutionnaliser socialement et à le transformer en sens commun.

\section{Une incapacité discursive}

Cette réduction obsessionnelle du débat sur l'axe sécuritaire est particulièrement dommageable dans des sociétés qui ne disposent d'aucun référentiel préexistant pour penser l'immigration. La comparaison avec le cas du Portugal est ici éclairante ${ }^{24}$. Sur la base de son expérience coloniale et des conditions de la décolonisation, cet État a en effet bâti des mécanismes de régulation politique et institutionnelle de la présence immigrée ${ }^{25}$. Il a pensé, dès sa Constitution (art. 7.4) puis dans ses lois d'immigration, les droits et les devoirs des ressortissants de ses anciens territoires d'outre-mer ${ }^{26}$. Si ces principes ne règlent pas tout, loin de là, et en tout cas pas les problèmes sociaux de l'immigration, ils constituent cependant un consensus politique solide sur le statut immigré et un efficace garde-fou contre les dérives néopopulistes. En effet, le statut politique privilégié accordé aux ressortissants des pays de langue portugaise, sous condition de réciprocité, en a fait des étrangers moins étrangers que d'autres : il aménage une forme de citoyenneté lusophone, qui va jusqu'à reconnaître le droit de vote actif et passif aux élections locales et le droit de vote à certains référendums aux immigrés brésiliens et cap-verdiens. Peu à peu, la

20. Commission internationale d'enquête sur les émeutes racistes de février 2000 en Andalousie, El Ejido, terre de non-droit, Limans, Forum civique européen, 2000. Voir aussi www.collectifs.net

21. Il existe en Espagne « une fraction de la société dure et souvent raciste, proche des cercles économiques profitant le plus du travail clandestin, qui conteste toute augmentation de l'immigration légale. Une des raisons principales de l'attaque raciste contre les immigrés était de créer un climat de terreur pour les pousser à partir et ainsi les empêcher d'être régularisés » (conclusion de El Ejido, terre de non-droit, op. cit.).

22. Loi organique 8/2000 modifiant la loi organique 4/2000 sur les droits et libertés des étrangers en Espagne et sur l'intégration sociale.

23. Voir la contribution de C. Barbosa sur la politique migratoire espagnole dans Politiques de l'Étranger, op. cit., ainsi que V. Pérez-Diaz, B. Alvarez-Miranda, C. Gonzalez-Enriquez, España ante la inmigración, Madrid, Fundacione La Caíxa, Coll. Estudios sociales 8, 2001

24. Le cas portugais n'est envisagé ici que pour sa valeur de révélateur quant aux conditions rendant possible une construction politique de la question migratoire. On n'entrera pas dans les longs développements que nécessiterait le traitement de ses autres caractéristiques en matière d'immigration, qui sont pourtant aussi importantes que sa spécificité politique. Sur le plan historique, il faudrait en effet pouvoir développer les ambiguités idéologiques du lusotropicalisme. Sur le plan de la sociologie économique, il faudrait tenir compte de la reprise récente de l'émigration portugaise vers les autres pays européens, qui complexifie quelque peu le débat, et de l'insertion singulière des immigrés aux deux extrêmes du marché du travail portugais. Voir M.I. Baganha, « A cada Sul o seu Norte : dinâmicas migratorias em Portugal », dans B. de Sousa Santos (org.), Globalização : fatalidade ou utopia, Porto, Afrontamento, 2001, pp. 135-159.

25. P. Dupraz, F. Vieira, « Immigration et modernité : le Portugal entre héritage colonial et intégration européenne », Pôle Sud, op. cit. ; F.L. Machado, «Etnicidade em Portugal. Contrastes e politização », Sociología. Problemas e práticas, 12, 1992 ; voir les contributions de F.L. Machado et de M. Marques avec N. Dias sur le Portugal dans Politiques de l'Étranger, op. cit. 26. Historiquement, « pour de bonnes ou de mauvaises raisons (tolérance, paternalisme des colonisateurs), les Africains des pays de langue portugaise, les Chinois de Macao, les Indiens de Goa ou les Maubères de Timor n'ont pas encore été rangés du côté de l'Autre, du point de vue national »(S. Lopes, « Le Portugal et ses immigrés », Migrations Sociétés, 4(19), 1992). 
société portugaise a fait l'expérience de la présence sociale et politique des immigrés lusophones, et cela lui a permis de rester relativement indifférente aux tentatives d'exploitation néopopuliste du thème par le Parti populaire lors de la dernière campagne électorale. Cependant, le consensus politique sur la question migratoire s'est bâti sur la représentation d'une communauté historique et linguistique, inspirée du lusotropicalisme, qui est désormais débordée par l'arrivée d'immigrés ukrainiens, pakistanais, chinois, indiens : le fait que la nouvelle loi portugaise sur l'immigration, votée en 2000, instaure des quotas de travailleurs immigrés par pays et lie le permis de séjour à l'existence d'un contrat de travail atteste déjà d'un changement du traitement de la question migratoire, qui se fait plus européenne que lusocentrique ; il sera donc intéressant d'observer la capacité de résistance du consensus portugais dans les années à venir, pris qu'il sera entre des processus d'intégration sociale inédits et des tentatives d'instrumentalisation néopopulistes.

Malgré ces incertitudes, ce cas montre que l'existence d'un référentiel fort sur l'identité de la nation et de l'État permet de borner le débat sur la présence étrangère dans des limites claires et saines. Au contraire, le débat peut dériver en Italie et en Espagne non seulement parce que les exploitations sécuritaires y sont centrales, mais aussi parce que la présence étrangère questionne des points aveugles des cultures politiques nationales : il n'y a pas en Italie de consensus fort sur les principes fondateurs de la Nation ni sur les règles essentielles de la puissance publique ; il y a en Espagne, autour de la question des autonomies, une dissension sur la (les) question(s) nationale(s), et de nombreuses disputes sur la légitimité des détenteurs de la puissance publique. La présence étrangère y révèle bien, en miroir, les incertitudes politiques. Elle pose en effet deux questions fondamentales : qu'il s'agisse d'intégrer ou d'exclure l'Étranger, au nom de quelle communauté nationale le proclame-t-on ? S'il faut déterminer une citoyenneté singulière pour l'immigré, sur quelles normes publiques va-t-on l'appuyer ?

Les États-nations incapables de répondre à ces interrogations n'ont qu'une alternative : soit ils se rabattent sur le plus petit dénominateur commun qui consiste en un mélange des principes minimaux de Schengen d'une part, et des valeurs universalistes catholiques, principale source d'inspiration des mobilisations en faveur des immigrés ${ }^{27}$, d'autre part ; soit ils se laissent aller aux pires dérives sécuritaires. Ayant glissé sur cette voie, l'Italie et l'Espagne sont doublement dans une impasse.

Impasse sociologique tout d'abord : les agences politiques et les médias qui ont tenu des discours sécuritaires ont renforcé la plausibilité des réactions de rejet et ont donné une légitimation politique à la xénophobie. En Italie et en Espagne, non seulement les incidents xénophobes se multiplient mais en outre ils se manifestent sans complexes. Les incidents des premières années étaient fondés sur une perception de la marginalité sociale des immigrés : selon les convictions, celle-ci générait soit de la solidarité militante avec un malheur social, soit du rejet conformiste face à un 
danger social. La figure de l'immigré était soit celle d'une victime soit celle d'un délinquant. Désormais, l'intolérance, parce qu'elle a été construite et légitimée sur le mode sécuritaire, se justifie aussi par le danger représenté par la différence culturelle. Plus l'immigré est culturellement différent, plus il représente un péril identitaire : inévitablement les musulmans, qui sont nombreux en raison des flux d'arrivées en provenance du Maghreb et des Balkans, sont les plus stigmatisés ${ }^{28}$. Tous les observateurs s'accordent ainsi à dire que l'hostilité envers les musulmans à la suite des événements du 11 septembre 2001 n'a fait qu'aggraver un rejet déjà installé et en grande partie politiquement construit : ce n'est pas un hasard si, alors qu'hier les incidents xénophobes éclataient à propos de l'insalubrité d'un quartier immigré ou des supposés trafics illicites des groupes immigrés, aujourd'hui les conflits se focalisent sur la construction des mosquées et des centres culturels islamiques. La genèse politique de cette stigmatisation des musulmans est particulièrement claire en Italie où les incidents de ce type se localisent pour la plupart dans les zones d'influence politique de la Ligue, c'est-à-dire dans le nord de la péninsule. C'est aussi le pays où politiques et leaders d'opinion ont le plus instrumentalisé la méfiance vis-à-vis de l'islam : rappelons les fâcheuses déclarations de Silvio Berlusconi à propos de la « supériorité » de la culture occidentale sur la culture musulmane le 26 septembre 2001, à Berlin, et le pamphlet raciste de la journaliste Oriana Fallaci, quatorze pages publiées par le Corriere della Sera le 29 septembre, avant de devenir un livre à succès. Sur ce fond de débat très malsain, la Ligue a continué à agiter le fantasme de l'invasion musulmane, particulièrement utile dans la période fin 2001-début 2002 où était en discussion la nouvelle loi sur l'immigration, dont Umberto Bossi (désormais convaincu que ses revendications de « dévolution » en faveur des régions septentrionales sont vaines) avait fait son principal cheval de bataille. La chronique des prises de position de la Ligue est révélatrice : de la marche des militants à Lodi contre la construction de mosquées (octobre 2000), en passant par le rapport des sénateurs de la Ligue sur « les avancées de l'islam en Italie » (fin 2001), jusqu'à la demande récente adressée au ministère de l'Intérieur par le vice-président de la Chambre des députés, membre du même parti, de fermer mosquées et centres culturels islamiques et de réduire au maximum les visas pour les étrangers musulmans (août 2002).

\footnotetext{
27. Sur la fonction des nouvelles régulations de la question migratoire par les ONG catholiques, voir la contribution de X. Itçaina et A. Dorangricchia dans Politiques de l'Étranger, op. cit.

28. Les musulmans sont estimés à 621000 personnes en Italie (Fondazione Migrantes, 2002) où la majorité des immigrés sont chrétiens (48 \%, contre 37 \% de musulmans)(Caritas Italia, 2002). Les chiffres sont très incertains en Espagne où certains chercheurs avancent le nombre de 300 000, alors que les associations musulmanes annoncent 450000 personnes (J. Moreras, Musulmanes en Barcelona, Barcelone, CIDOB edicions, 1999). Au Portugal, ils sont environ 30 000, pour la plupart de nationalité portugaise (Observatoire européen des phénomènes racistes et xénophobes, 2001).
} 
La montée du rejet des immigrés musulmans est plus complexe en Espagne, où le gouvernement Aznar est trop pragmatique pour laisser se développer les mêmes outrances, et aussi parce qu'il n'y existe pas de partis, sinon ultra-minoritaires, susceptibles de développer des thèses extrémistes : le référent démocratique, hérité de la transition espagnole, entretient encore quelques tabous. Mais le message sécuritaire a, là aussi, ouvert une brèche dans le consensus démocratique, où s'engouffrent des réactions sociales d'intolérance : refus des lieux de culte musulmans, plus grande difficultés d'intégration sociale pour les immigrés de confession musulmane à propos du logement, du travail ou de l'école ${ }^{29}$. De façon très révélatrice, ces réactions sont codées en termes identitaires : malgré les protestations de la hiérarchie et des ONG catholiques en faveur de la coexistence harmonieuse des religions, une partie de la société espagnole semble ne se projeter que dans son héritage catholique et craint le retour, venu de son lointain passé, des Moros : la violence des événements d'El Ejido, souvent justifiée par le trop grand écart culturel entre Espagnols et Marocains, a ouvert la boîte de Pandore de l'intolérance identitaire. De même, la Communauté autonome où résident le plus d'immigrés, la Catalogne, qui a toujours défendu l'idéologie du « creuset catalan » dans lequel viendraient se fondre tous ceux qui acceptent de vivre en Catalans ${ }^{30}$, voit-elle son paradigme d'intégration mis en crise : celui-ci, essentiellement fondé sur l'acceptation de la langue et de la culture catalanes par des migrants espagnols, se trouve démuni pour penser la différence religieuse et culturelle des extracommunautaires. Là aussi, malgré la politique officielle de tolérance religieuse, de nombreux comités se sont créés pour protester contre la construction de mosquées ${ }^{31}$ ou le trop grand nombre d'enfants de familles musulmanes dans les écoles. Une rumeur insistante court donc dans ces deux pays, selon laquelle il vaudrait mieux ne laisser entrer que des immigrés chrétiens : comment ces sociétés pourront-elles, désormais, retrouver un débat constructif sur des immigrations que l'ont sait inéluctables?

À cette impasse sociologique s'en ajoute une autre, plus politique. Les gouvernements qui ont prôné le choix sécuritaire ne sont pas en mesure de réaliser ce qu'ils avancent. L'épreuve de la réalité vient très vite : il n'est pas possible de contrôler véritablement des frontières, d'autant moins dans la situation géostratégique de ces zones ; il n'est pas envisageable de rapatrier les immigrés clandestins en masse ; l'économie, formelle ou informelle, ne peut pas se passer de la main-d'œuvre immigrée ; la restriction des entrées légales et l'absence de droits précis pour les immigrés entretiennent le cercle vicieux de la clandestinité. Tout choix sécuritaire autoritaire, c'est-à-dire fonctionnant exclusivement sur la thématique de la protection du territoire, ne peut que voir très vite dévoilée son incapacité32. Confrontés à cette perspective, les gouvernements espagnol et italien n'ont pas tardé à tenter, de concert, de se défausser sur l'UE des contraintes sécuritaires qu'ils ont eux-mêmes construites. 


\section{Solitude diplomatique}

La montée des pratiques sécuritaires, souvent unilatérales, et des manifestations xénophobes, notamment anti-musulmanes, assombrit les rapports de ces États avec les pays du pourtour méditerranéen : elle défigure leurs rapports de partenaires traditionnels, affaiblit leur rôle de médiateur dans les grands conflits méditerranéens. Comme le fut la théorie de l'immigration zéro prônée par les principaux pays d'immigration en Europe dans les années soixante-dix, l'actuelle crispation sécuritaire des pays sud-européens est en effet contre-productive dans la perception des responsables politiques des pays d'origine : ces prises de position sont explicitement accusées d'avoir favorisé l'organisation de filières clandestines qui exploitent la misère des candidats à l'émigration, et d'avoir entraîné une perpétuation des communautés immigrées à l'étranger ${ }^{33}$. Le caractère unilatéral des processus de décision est d'autant plus mal vécu que les pays partenaires sont jaloux de leur souveraineté, comme en attestent tous les jours les difficultés diplomatiques avec le Maroc.

L'inefficacité du multilatéralisme en la matière (absence d'une politique migratoire communautaire et réduction du partenariat euro-méditerranéen à des déclarations de bonnes intentions en matière de circulation des personnes) a laissé ces États d'autant plus démunis : dans les débats intérieurs sur la politique migratoire, la dimension euro-méditerranéenne n'est jamais évoquée. Dans un sursaut de réalisme politique, ils se sont donc particulièrement investis dans la recherche d'accords bilatéraux avec les pays de la rive Sud, dont le premier a été l'accord de réadmission entre l'Espagne et le Maroc signé en 1992, suivi en 1994 de l'accord de réadmission entre la France et l'Algérie. L'Italie a signé, entre 1996 et 2000, vingtdeux accords de réadmission concernant les ressortissants des pays signataires

29. Voir les rapports de l'Observatoire européen des phénomènes racistes et xénophobes sur les réactions anti-islamiques récentes dans les différents pays européens, www.eumc.eu.int/publications

30. C. Barbosa, « Le "creuset catalan”. Construction nationalitaire et capacité d'intégration », Pôle Sud, op. cit.

31. À Premià del Mar par exemple. Voir La Vanguardia tout au long du mois de mai 2002.

32. Ainsi que son impossibilité à s'adapter aux nouvelles caractéristiques des migrations contemporaines, marquées par des mouvements pendulaires entre pays de départ et pays d'arrivée, et par une forte tendance à la circulation transnationale. 33. «Depuis la fin des années soixante-dix, la seule approche politique en matière d'immigration a été l'idée d'immigration zéro. L'immigration zéro n'est pas une politique, c'est une non-politique qui a laissé des forces non organisées (j'entends par là non organisées sur le plan institutionnel mais actives de fait) s'en occuper directement, hors de la loi et des règlements, hors des normes régissant les échanges entre êtres humains et la circulation des personnes [...], et agir sur le marché de la main-d'œuvre. Contrairement à ce qu'on aurait pu attendre, ceci n'a pas entraîné une croissance zéro des flux migratoires, mais bien au contraire une augmentation de l'immigration. J'en suis témoin, je viens d'un pays créateur d'immigration. À la fin des années soixante-dix, mon pays avait 800000 ressortissants à l'extérieur, aujourd'hui ils sont plus de deux millions, dans le cadre de la non-politique de l'immigration », Intervention du ministre marocain du Développement social au congrès « Migrazioni. Scenari per il XXI secolo », Agenzia romana per la preparazione del Giubileo, Rome, juillet 2000, cité par G. Sciortino, La rotta di Enea. Relazioni euromediterranee e migrazioni, 2001, www.cespi.it 
aussi bien que ceux des pays tiers ayant transité par les territoires de ces derniers ${ }^{34}$. L'Espagne en a passé une dizaine ${ }^{35}$. Cependant il ne s'agit là que d'une technique diplomatique bilatérale, censée compléter les dispositifs de contrôle des frontières : il est patent que cette méthode ne produit des effets que lorsqu'elle s'accompagne au minimum d'un accord sur un quota d'entrées légales accordé au pays partenaire, ou mieux d'incitations économiques négociées parallèlement ${ }^{36}$. C'est ainsi que l'action diplomatique du gouvernement de centre-gauche en Italie, entre 1996 et 2000, a été considérée comme novatrice en la matière, étant donné le traitement privilégié accordé à la Tunisie, au Maroc et à l'Albanie, qui prévoit des quotas annuels d'entrées pour leurs ressortissants et des opérations de développement sur place (aussi bien au niveau des États qu'au travers des échanges de coopération décentralisée, très nombreux en Italie ${ }^{37}$ ). L'Espagne s'est engagée sur la même voie à partir de 2001 en passant des accords de ce type avec le Maroc, l'Équateur, la Colombie, la Roumanie, qui organisent des quotas d'entrées et des conditions strictes de travail saisonnier ${ }^{38}$.

Ce retour au bilatéralisme est une avancée par rapport au paradigme du marché (l'illusion que, par un effet mécanique, les investissements financiers et commerciaux devaient entraîner le ralentissement des flux migratoires) qui semble avoir paralysé le processus euro-méditerranéen sur ces questions. Cependant il se heurte lui aussi à la forte dissymétrie des intérêts sur les deux rives. De ce point de vue, les difficultés récurrentes rencontrées avec le Maroc sont révélatrices : en Italie, les quotas d'entrées pour les ressortissants marocains ont été réduits en 2001, manifestation de la déception italienne dans la collaboration avec le Maroc en matière de réadmission ; avec l'Espagne, la négociation de l'accord de 2001 s'est spectaculairement bloquée (rappel de l'ambassadeur marocain à Madrid en octobre 2001), sur des désaccords qui portent autant sur les questions migratoires que sur les droits de pêche. Il est à craindre que cette politique de quotas de maind'œuvre, mise en place par les pays européens du Sud comme principal instrument de contrôle de l'immigration, ne limite très vite leur politique à un paradigme productiviste, étrange réminiscence de celui qui dominait en Europe de l'Ouest dans les années soixante. Cette interprétation, récente dans les pays sud-européens, est en effet en train de devenir objet de débat : la gestion économique de l'immigration y est discutée ; les organisations patronales, arguant des nécessités de l'économie nationale, prennent position pour une augmentation des quotas ; certaines régions italiennes et Communautés autonomes espagnoles réclament le droit d'établir leurs propres quotas. Si, parallèlement au répertoire de la forteresse et à celui de l'hospitalité caritative qui structurent le débat jusqu'ici, s'imposait un répertoire productiviste, on sortirait du tout sécuritaire par une bien petite porte, celle du calcul économique.

En réalité, cette timide tentative de négociation elle-même achoppe déjà sur la poli- 
tisation de la question migratoire. La nouvelle loi italienne sur l'immigration votée en août 2002 ne prévoit plus de quotas d'entrées préférentiels ni de programmation des flux : on peut donc craindre que la petite avancée des accords bilatéraux ne tombe en désuétude ${ }^{39}$. Mais surtout les gouvernements italien et espagnol actuels sont parfaitement d'accord sur une politique autoritaire et unilatérale de l'immigration. Peinant à en gérer eux-mêmes les conséquences, ils se sont alliés pour tenter d'imposer à l'Union européenne une politique de conditionnalité « négative » : ou les partenaires bloquaient les flux d'immigration illégale (contrôle renforcé des frontières et réadmissions efficientes) ou l'UE annulait aides financières et régimes préférentiels. Cette option radicale et peu réaliste a fait long feu au niveau communautaire, même si elle a contribué à recentrer la politique communautaire sur le paradigme sécuritaire : depuis le Conseil européen de Séville (juin 2002), le management des frontières extérieures par les instances communautaires est fortement réactivé.

Le cercle vicieux sécuritaire s'est donc enroulé en une boucle infernale : lancé par les accords intergouvernementaux de Schengen, repris et amplifié au gré des tendances sécuritaires qui agitent certains États, il s'est refermé sur le niveau communautaire. Pas plus celui-ci que les États ne peuvent échapper au piège de ses effets pervers : face au caractère inéluctable de l'immigration, plus l'interprétation sécuritaire est exclusive, plus il faudra, pour la surmonter, passer par la grande porte d'un paradigme politique fort. Porte que tentent d'entrouvrir certains partis et mouvements nationaux, l'Assemblée européenne ${ }^{40}$ et le Conseil européen de Feira ${ }^{41}$, lorsqu'ils réclament l'ouverture d'une réflexion générale sur une citoyenneté immigrée, une citoyenneté détachée de la nationalité : quel contrat civique

\footnotetext{
34. Slovénie, Macédoine, Roumanie, Géorgie, Hongrie, Lituanie, Lettonie, Estonie, Yougoslavie, Croatie, France, Autriche, Albanie, Bulgarie, Maroc, Slovaquie, Tunisie, Suisse, Grèce, Espagne, Algérie, Nigeria (www.cittadinolex.kataweb.it)

35. France, Maroc, Pologne, Portugal, Roumanie, Bulgarie, Italie, Lettonie, Estonie, Slovaquie. (J. Badosa, J. Subirats, Alone against the Danger: The Role of the Ministry of Interior in Spanish Migration Policy and the Lack of Governance Model, communication au Congrès de l'ECPR, Turin, avril 2002).

36. MigraCtion Europa (Bollettino trimestrale di analisi delle politiche migratorie in Europa), 3, 2002, www.cespi.it ; F. Pastore, «L'obbligo di riammissione in diritto internazionale : sviluppi recenti », Rivista di diritto internazionale, 4, 1998.

37. A. Stocchiero (dir.), Dossier Politiche migratorie e di cooperazione nel Mediterraneo, octobre 2001, www.cespi.it

38. Les institutions communautaires ont pris le même chemin en prévoyant dans les accords euro-méditerranéens d'association des incitations à la réadmission et à la coopération au développement (accord avec la Tunisie en 1995, entré en vigueur en 1998 ; accord avec le Maroc en 1996, entré en vigueur en 2000) ; en outre, depuis l'entrée en vigueur du traité d'Amsterdam, les institutions communautaires ont compétence pour signer des accords de réadmission communautaire qui devraient venir compléter les accords bilatéraux.

39. Je dois ces remarques sur la situation récente à G. Sciortino ; voir aussi G. Sciortino, La rotta di Enea, op. cit., et sa contribution sur la politique migratoire européenne dans Politiques de l'Étranger, op. cit. Ajoutons que la loi italienne actuelle annule la possibilité de sponsorisation des immigrés (c'est-à-dire le fait de se porter garant de l'existence d'un contrat de travail et d'un logement au moment de la demande de visa) par des organisations collectives et conditionne l'obtention d'un visa à l'existence antérieure d'un contrat de travail.

40. « Recommandations sur la politique méditerranéenne de l'UE », Assemblée européenne, mars 1999.

41. «Stratégie commune de l'UE pour la région méditerranéenne », Conseil européen, Feira, juin 2000.
} 
est-il envisageable pour une reconnaissance des droits, pour des modalités de représentation, pour les conditions d'accès au droit de vote, pour les modalités d'acquisition de la nationalité ou pour la protection du droit d'aller et venir ${ }^{42}$ ? Ce n'est qu'en répondant enfin à ces questions que l'on pourrait sereinement poser les principes des conditions d'entrée et de séjour sur le territoire de l'Union européenne. Or les États sud-européens qui ont choisi la voie sécuritaire exacerbée se sont dramatiquement éloignés de cette perspective politique, au sens noble du terme : ils ont contribué à forger des représentations menaçantes de l'immigration, ils ont mis à mal les longs et fragiles processus de l'intégration sociale, ils ont spéculé sur l'incertitude au lieu d'encourager à la responsabilité, et ils semblent donc aujourd'hui, politiquement, autant dos au mur que dos à la mer.

Évelyne Ritaine est chercheur au CERVL - Pouvoir, Action publique, Territoire, Institut d'études politiques de Bordeaux. E-mail : e.ritaine@sciencespobordeaux.fr

42. É. Balibar, Nous, citoyens d'Europe? Les frontières, l'État, le peuple, Paris, La Découverte, 2001, en particulier le chap. 10, «L'Europe des citoyens ». 